\title{
The advantage of factorial mating under selection is uncovered by deterministically predicted rates of inbreeding
}

\author{
Anders Christian SøRENSEN ${ }^{\mathrm{a}, \mathrm{b}, \mathrm{c} *}$, Peer BERG ${ }^{\mathrm{b}}$, \\ John A. WOOLLIAMS ${ }^{\mathrm{a}}$ \\ ${ }^{\text {a }}$ Roslin Institute (Edinburgh), Roslin, Midlothian, EH25 9PS, UK \\ ${ }^{\mathrm{b}}$ Department of Animal Breeding and Genetics, Danish Institute of Agricultural Science, \\ PO Box 50, 8830 Tjele, Denmark \\ ${ }^{\mathrm{c}}$ Department of Large Animal Sciences, Royal Veterinary and Agricultural University, \\ Ridebanevej 12, 1870 Frederiksberg C, Denmark
}

(Received 14 October 2003; accepted 18 August 2004)

\begin{abstract}
Rates of inbreeding $(\Delta F)$ in selected populations were predicted using the framework of long-term genetic contributions and validated against stochastic simulations. Deterministic predictions decomposed $\Delta F$ into four components due to: finite population size, directional selection, covariance of genetic contribution of mates, and deviation of variance of family size from that expected from a Poisson distribution. Factorial (FM) and hierarchical (HM) mating systems were compared under mass and sib-index selection. Prediction errors were in most cases for $\Delta F$ less than $10 \%$ and for rate of gain less than $5 \% . \Delta F$ was higher with index than mass selection. $\Delta F$ was lower with FM than HM in all cases except random selection. FM reduced the variance of the average breeding value of the mates of an individual. This reduced the impact of the covariance of contributions of mates on $\Delta F$. Thus, contributions of mates were less correlated with FM than HM, causing smaller deviations of converged contributions from the optimum contributions. With index selection, FM also caused a smaller variance of number of offspring selected from each parent. This reduced variance of family size reduced $\Delta F$ further. FM increases the flexibility in breeding schemes for achieving the optimum genetic contributions.
\end{abstract}

mating system / inbreeding / selection / prediction / genetic contribution

\section{INTRODUCTION}

Simulated dairy cattle breeding schemes based on selection at an early age, combining family information with technologies affecting the reproductive capacity of females, resulted in extremely high rates of inbreeding [12,14].

*Corresponding author: AndersC.Sorensen@agrsci.dk 
This occurred because all male offspring from the best family were selected together. Therefore, some restrictions on selection were imposed so that e.g. only one male could be selected from each full-sib family [11]. This, however, decreased the selection intensity, because males with lower predicted breeding values were selected. Woolliams [15] proposed the application of a mating strategy, factorial mating, that gives a different population structure compared to hierarchical mating by reducing the size of full-sib families while increasing the number of half sibs. Factorial mating is a random mating system, where parents of both sexes are mated to more than one of the opposite sex. This is in contrast to hierarchical mating schemes, where females are only mated to one male, but one male can be mated to several females. Factorial mating, therefore, results in a smaller risk of selecting many animals from the same full-sib family. Consequently, factorial mating is expected to decrease the variance of family sizes after selection and, thus, to result in a smaller rate of inbreeding. When selection is directional, factorial mating has been shown to increase the rate of gain with a small reduction in the rate of inbreeding relative to hierarchical mating $[12,14]$. However, stochastic simulations do not uncover the mechanisms by which factorial mating reduces inbreeding. An alternative approach is to model the selection and mating process deterministically, by setting up a series of prediction equations, revealing how mating structures influence the rate of inbreeding. Such a framework for predicting both rates of gain and rates of inbreeding under selection has been developed by Woolliams and co-workers using long-term genetic contributions [16, 18]. So far, this framework has not been applied to mating systems other than hierarchical mating.

This study shows that it is possible, by deterministically predicting the rate of inbreeding, to quantify in what way, and why, factorial mating reduces the rate of inbreeding relative to hierarchical mating in populations under selection. In order to predict the rate of inbreeding, the concept of long-term genetic contributions was used and the framework was extended and validated to account for factorial mating.

\section{MATERIALS AND METHODS}

The basic assumptions underlying this study were those of a breeding program in equilibrium, i.e. a population with a stable genetic variance, and with a trait that can be modelled by the infinitesimal model [8]. Stable genetic parameters were obtained by iterating on the recurrence relationships of Bulmer [5], 
while any effect of inbreeding on the genetic variance was ignored in order to avoid interactions between the rate of gain and the rate of inbreeding.

Two mating designs were explored in this study. Hierarchical mating, where dams are nested within sires, i.e. a dam is mated to a single sire and so has a mating ratio, $d_{f}=1$, whereas sires can be mated to more than one dam, $d_{m} \geq 1$. Under factorial mating, on the other hand, both sires and dams are mated to more than one of the opposite sex, i.e. a cross-classified design, where both $d_{m}$ and $d_{f}$ are $>1$. When these two mating schemes are compared with the same number of offspring per sire and per dam, they result in different family structures. Hierarchical mating results in large full-sib families and a group of paternal half-sibs. Factorial mating results in small full-sib families, more paternal half-sibs, and also a group of maternal half-sibs. In this study, both mating systems were implemented using random mating, in the sense that there is no expected covariance between any characters of mates.

Selection was based on an index that weighs information of the candidates own performance with the performance of full-sibs, paternal half-sibs, and maternal half-sibs [7]. The index for the $k$ th offspring of the $i$ th sire and $j$ th dam is:

$$
I_{i j k}=c_{1}\left(P_{i j k}-\bar{P}_{i j}\right)+c_{2}\left(\bar{P}_{i j}-\bar{P}_{i \bullet}-\bar{P}_{\bullet j}+\bar{P}_{\bullet \bullet}\right)+c_{3}\left(\bar{P}_{i \bullet}-\bar{P}_{\bullet \bullet}\right)+c_{4}\left(\bar{P}_{\bullet j}-\bar{P}_{\bullet \bullet}\right)
$$

where $P_{i j k}$ is the performance of the individual itself, $\bar{P}_{i j}$ is the average performance of the full-sib family, $\bar{P}_{i \bullet}$ is the average performance of the paternal half-sib family of sire $i, \bar{P}_{\bullet j}$ is the average performance of the maternal half-sib family of dam $j$, and $\bar{P}_{\bullet}$ is the population mean. The first information source is independent of the others, but the remaining sources are mutually dependent unless $d_{f}=1$. In this case, which is hierarchical mating, $\bar{P}_{\bullet j}=\bar{P}_{i j}$, and the index reduces to a form comparable to the sib-index of Wray and Hill [20] and Wray et al. [22].

\subsection{Setting up the model}

The rates of gain and inbreeding were predicted by modelling the selection and mating process. Thus, the mechanisms generating gain and inbreeding were revealed in this model. The basis of the model is the concept of long-term genetic contributions first introduced by James and McBride [9].

\subsubsection{Expected long-term genetic contributions}

The long-term genetic contribution of an ancestor in a remote generation is the proportion of genes in the present generation, which have been derived 
directly from the ancestor [18]. Wray and Thompson [21] derived a relationship between the realised genetic contribution and the rate of inbreeding, and Woolliams and Thompson [17] derived an analogous expression for the relationship between the realised genetic contribution and the rate of gain.

Long-term genetic contributions can be predicted conditional on selective advantages of individuals, i.e. factors influencing the success of an animal measured as the number of offspring selected [18]. Thus, predicted genetic contributions model the expected transmission of selective advantages from parent to offspring.

The selective advantages considered in this study were (1) the breeding value of the individual itself, and (2) the average breeding value of the mates of the individual. The second selective advantage takes account of the fact that the probability of having offspring selected depends on the breeding value of the other parent. The expected long-term genetic contribution, $r_{i(q)}$, of individual $i$ of $\operatorname{sex} q$ were calculated conditional on these selective advantages using a linear model:

$$
E\left[r_{i(q)} \mid i \text { is selected }\right]=\mu_{i}(q)=\alpha_{q}+\beta_{q, 1}\left(s_{i(q), 1}-\bar{s}_{q, 1}\right)+\beta_{q, 2}\left(s_{i(q), 2}-\bar{s}_{q, 2}\right)
$$

where $\left(s_{i(q), 1}-\bar{s}_{q, 1}\right)$ is the true breeding value of the individual itself as a deviance from the mean breeding value of parents of sex $q$, and $\left(s_{i(q), 2}-\bar{s}_{q, 2}\right)$ is the average true breeding value of the mates of the individual as a deviance from the mean.

The model requires intercept and regression parameters $\left(\alpha_{q}, \beta_{q, 1}, \beta_{q, 2}\right)$ to be predicted. The intercept terms are specific for each sex and are easily predicted for non-overlapping generations, as they only depend on the number of parents of each sex, $N_{q}$, and are independent of the selection process. Thus:

$$
\alpha_{q}=\frac{1}{2 N_{q}}
$$

A general solution for predicting the regression terms was presented by Woolliams et al. [18]. The regressions $\left(\beta_{q, 1}, \beta_{q, 2}\right)$ were predicted using two linear models to describe the inheritance of the selective advantages. First, the number of selected offspring as a function of the parent's selective advantages. And second, the selective advantages of selected offspring as a function of the parent's selective advantages. The details of the predictions are presented in Appendix A. 


\subsubsection{Predicted rates of genetic gain}

The expected rates of genetic progress were calculated from the expected long-term genetic contributions, $\mu_{i(q)}$, following Woolliams et al. [18]:

$$
\begin{aligned}
E\left[\Delta G_{e q}\right] & =\sum_{q=m, f} N_{q} \cdot E\left[\mu_{i(q)} \cdot a_{i(q)} \mid i \text { is selected }\right] \\
& =\sum_{q=m, f} N_{q} \cdot\left(\begin{array}{c}
\alpha_{q} E\left[a_{i(q)} \mid i \text { is selected }\right] \\
+\beta_{q, 1} \operatorname{cov}\left(s_{i(q), 1}-\bar{s}_{q, 1} ; a_{i(q)}\right)
\end{array}\right)
\end{aligned}
$$

where $a_{i(q)}$ is the Mendelian sampling term of ancestor $i$ of sex $q$, and $N_{q}$ is the number of ancestors of sex $q$. The term in $\beta_{q, 2}$ has disappeared, because the covariance between the Mendelian sampling term of an individual and the breeding values of the mates is zero under random mating. The expectations and covariances in (2) are given in Appendix B for the scenarios considered in this study.

\subsubsection{Predicted rates of inbreeding}

The rate of inbreeding is also a function of the long-term contributions of ancestors, $r_{i},[16,21]$ :

$$
\Delta F \approx \frac{1}{4} E\left[\sum_{i} r_{i}^{2}\right] .
$$

This is a simplified version assuming random mating. In a prediction problem, the contributions are not observed, so they are replaced with expectations:

$$
E[\Delta F] \approx \frac{1}{4} \sum_{i} E\left[r_{i}^{2}\right]=\frac{1}{4} \sum_{i}\left[E\left[r_{i}\right]^{2}+\operatorname{Var}\left[r_{i} \mid \text { selective advantages }\right]\right] .
$$

If family sizes follow a Poisson distribution, family size meaning the number of selected offspring of a specific parent, then the variance is determined by the mean. Any deviation from the expected variance is modelled in a term, $\delta$, which is composed of two parts: the deviation of family size from Poisson and the deviation of genetic contributions from the linear regression in (1). Then (3) becomes:

$$
E[\Delta F] \approx \frac{1}{2} \sum_{i}\left[\mu_{i}^{2}+\frac{1}{4} \delta_{i}\right]
$$


and using (1) gives with some modifications:

$$
\begin{aligned}
& E\left[\Delta F_{e q}\right] \\
& =\left(1+2 E\left[\Delta F_{e q}\right]\right)\left[\frac{1}{2} \sum_{q=m, f} N_{q} \cdot E\left[\mu_{i(q)}^{2} \mid i \text { is selected }\right]+\frac{1}{8} \sum_{q=m, f} N_{q} \delta_{q}\right] \\
& =\left(1+2 E\left[\Delta F_{e q}\right]\right) \\
& \quad\left[\frac{1}{2} \sum_{q=m, f} N_{q} \alpha_{q}^{2}+\frac{1}{2} \sum_{q=m, f} N_{q} \beta_{q, 1}^{2} \operatorname{Var}\left(s_{i(q), 1}-\bar{s}_{q, 1}\right)\right. \\
& \left.+\frac{1}{2} \sum_{q=m, f} N_{q} \beta_{q, 2}^{2} \operatorname{Var}\left(s_{i(q), 2}-\bar{s}_{q, 2}\right)+\frac{1}{8} \sum_{q=m, f} N_{q} \delta_{q}\right] .
\end{aligned}
$$

The first term is a second-order correction, because the expression inside the square brackets is expected to under-predict the rate of inbreeding by a fraction of two $\Delta F$ [16]. The correction arises from the derivation of the relationship between the rate of inbreeding and the long-term contribution. The first three terms inside the square brackets of (4) were derived assuming that size of families after selection follow a Poisson distribution [16]. The variances of the selective advantages are given in Appendix B.

The fourth term inside the square brackets in (4) includes $\delta_{q}$, which is the correction (hereafter called Poisson correction) due to deviations of the variance of family size from the variance expected under a Poisson distribution and deviations from the linear model (1). In this paper, family sizes are equal prior to selection, and follow a hypergeometrical distribution after selection [4]. For mass selection the hypergeometrical distribution was approximated by a binomial distribution [4].

The family structure of a population under factorial mating is different from under hierarchical mating. Under mass selection the difference has limited impact on the rate of inbreeding, and the binomial approximation for the Poisson correction can be adopted with very limited loss of precision [4]. Under index selection, correlations between indices of sibs can be very high, which increases the probability of co-selection of sibs. This has a significant impact on the rate of inbreeding, and is partly accounted for by the linear expression (1). However, the linear model is an approximation to a non-linear relationship between contributions and selective advantages [16]. The Poisson correction also accounts for the effect of this non-linearity on the rate of inbreeding, because the variance deviations are calculated as the part of the total variance not accounted for by the linear model (1). The Poisson correction was calculated 
using the approach by Woolliams and Bijma [16]. The detailed procedure for calculation of the corrections is given in Appendix C.

The four terms inside the square brackets of (4) are isolated terms each contributing to the rate of inbreeding and can be interpreted as follows.

I. The first term, containing the $\alpha$ 's, gives the rate of inbreeding had random selection been applied. This is the rate of inbreeding due to finite population size alone.

II. The second term, containing the regression on the first selective advantage, gives the contribution to the rate of inbreeding that arises due to the selection that generates genetic gain. This is called inbreeding due to directional selection.

III. The third term, containing the regression on the second selective advantage, gives the contribution to the rate of inbreeding that is due to the tying together of genetic contributions of the male and female of a mating pair without generating genetic gain. This is called inbreeding due to covariance between long-term genetic contributions of mates.

IV. The fourth term, the Poisson correction as explained earlier, is the correction for deviations from a Poisson distribution of the family sizes after selection and for deviations from linearity: the higher variances of family sizes and the larger deviations from linearity in the contributions, the larger correction.

The presented model was used to analyse differences between the two mating systems in the four terms contributing to the rate of inbreeding in equation (4).

\subsection{Validating the model}

The results from the deterministic model were compared to stochastic simulations in order to validate the predictions of rates of gain and inbreeding, as well as some of the different components in the predictions. The simulation program developed by Bijma et al. [4] was further developed to account for factorial mating. The breeding schemes were allowed five generations for achieving equilibrium in the parameters, and generation five was therefore used as the base population. An additional seven generations were simulated to allow contributions from ancestors in generation five to converge [2]. The rates of gain and inbreeding were measured as averages from generation 6 to 12 . The simulation program allowed calculations of empirical equilibrium genetic parameters, regression coefficients for the linear model and Poisson corrections for comparison to those predicted. This allowed a validation of single 
components of the prediction equation (4). E.g. the effect of imprecise predicted regression parameters on the precision of the predicted rate of inbreeding could be tested by using the predicted regression parameters in (4) and use simulated values for the rest of the terms.

Two different terms were calculated from the simulations in order to validate the predicted Poisson correction and assess the effect of non-linearity of the contributions relative to the selective advantages on the rate of inbreeding. The first term, $P C_{v}$, is a correction for deviation from Poisson variances alone. The second term, $P C_{v l}$, is a correction for both deviation from Poisson variances and deviation from linearity. This second term is a simulated term corresponding to the predicted Poisson correction.

A number of scenarios were used to validate the predictions. Four different population structures and sizes were explored with all combinations of 20 and 40 sires, and twice and four times as many dams as sires. In all populations, selection of parents was based on the index described above. The weights in the index were either (1) set equal to each other, which resulted in mass selection, or (2) calculated, conditional on the equilibrium parameters, using standard selection index theory in order to maximise the correlation between the true breeding value and the index, i.e. so-called optimum weights. The traits investigated ranged in heritabilities from 0.01 to 0.99 . Hierarchical mating $\left(d_{m}=2\right.$ or $\left.4, d_{f}=1\right)$ and factorial mating with a female mating ratio of four $\left(d_{m}=8\right.$ or $\left.16, d_{f}=4\right)$ were applied. Under hierarchical mating litter size was eight, i.e. four of each sex, and under factorial mating litter size was two, i.e. one of each sex. Consequently, the number of selection candidates and, therefore selected proportions, were the same under the two mating schemes.

\section{RESULTS}

\subsection{Mechanisms generating inbreeding}

The results presented in this section were derived from the deterministic model described under Materials and Methods. Therefore, no standard errors are attached to the presentation of the results and differences.

\subsubsection{Effect of heritability and selection criterion}

The rate of inbreeding depended on the heritability of the trait under selection, the selection criteria used, and the mating scheme in a complex manner for a given size of breeding scheme (Fig. 1). With classical selection indices, 


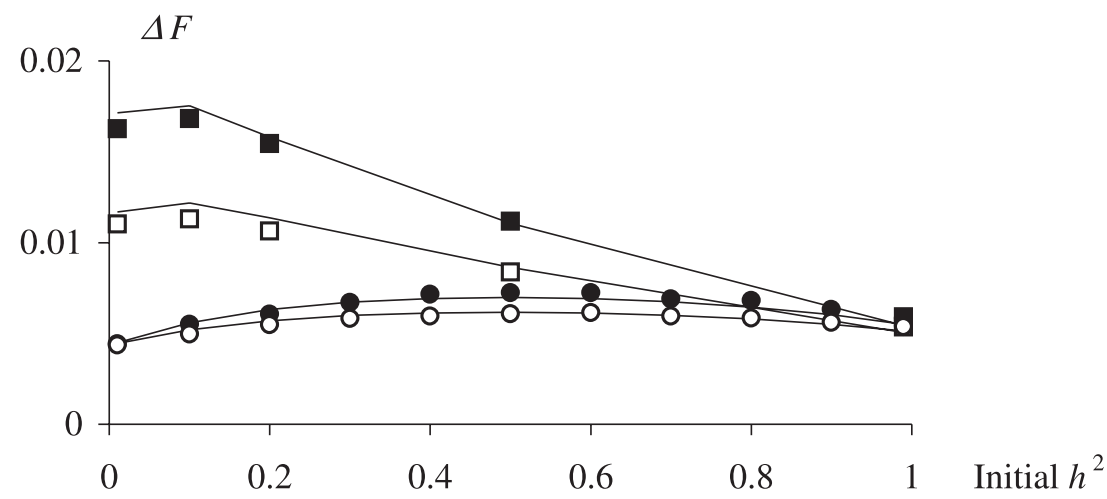

Figure 1. Predicted (lines) and simulated (symbols) rates of inbreeding $(\Delta F)$ in a scheme with 40 sires and 80 dams selected on phenotype (circles) or on sib-index (squares) and mated hierarchically (filled symbols) or factorially (open symbols) for a range of initial heritabilities $\left(h^{2}\right)$.

the rate of inbreeding was highest for low heritabilities and declined as heritability tends to 1 , but for mass selection the rate of inbreeding was highest for intermediate heritabilities. The classical index selection produced greater rates of inbreeding than mass selection (compared using the same mating system) except when heritability is 1 , when the two selection criteria are equivalent.

\subsubsection{Difference between mating systems}

There was no difference between the rates of inbreeding in the two mating schemes under random selection, which was approximated by mass selection for a trait with a heritability of 0.01 , as shown by both the simulated and predicted values in Figure 1. The predictions captured this property of factorial mating, because the variances of the selective advantages are zero under random selection. The finding that hierarchical and factorial mating resulted in the same rate of inbreeding under random selection is in accordance with the results of Woolliams [15]. This suggests that the looser tying together of contribution of mates under factorial mating is important, only when selection acts upon the contributions in attempting to increase the contribution of the better than average ancestors while decreasing contributions of other ancestors.

For low heritabilities $(\leq 0.2)$, factorial mating was very advantageous under index selection. The reasons for the differences between factorial and hierarchical mating were revealed by deterministic predictions of rates of inbreeding. Several factors contributed to a reduced rate of inbreeding with factorial 
mating under index selection. These were highlighted by the decomposition of equation (4) described previously.

The contributions to the rate of inbreeding from directional selection, covariance between mates, and the Poisson correction were smaller under factorial mating than under hierarchical mating (Fig. 2). The term due to directional selection was reduced between 0 and $30 \%$, with larger reductions for lower heritabilities, due to a lower accuracy of the selection criteria under factorial mating (the impact on genetic gain will be addressed in Sect. 3.2.2). The term due to covariance between mates was reduced due to contributions from females being less correlated to contributions from males under factorial mating. The Poisson correction was smaller under factorial mating due to a smaller variance of family sizes and smaller deviations from linearity in the relationship between contributions and selective advantages. Together, these factors contributed to a considerably lower rate of inbreeding with factorial mating relative to hierarchical mating, when selection was based on a sib-index.

\subsubsection{Non-linearity explained by the Poisson correction}

The impact of the deviations from linearity on the rate of inbreeding was assessed by comparing $P C_{v}$ and $P C_{v l}$ relative to the rate of inbreeding (Fig. 3). For cases with intermediate heritabilities under index selection disregarding the non-linearity would result in an under-rating of the rate of inbreeding by up $20 \%$. The degree of non-linearity was larger for hierarchical than for factorial mating. The amount of non-linearity in the relationship between contributions and selective advantages depended also on the heritability. For a heritability of 0.01 there was hardly any non-linearity, but the amount of non-linearity increased with increasing heritability up to moderate heritabilities. For the extreme heritability of 0.99 the amount of non-linearity was again smaller.

\subsubsection{Mass selection}

The predictions revealed that, under mass selection, the only differences between the mating schemes was that the variance of the second selective advantage was reduced under factorial mating for both males and females. With a female mating ratio of four, the variance was reduced by 77 to $79 \%$ compared to hierarchical mating (results not shown) giving a smaller contribution from the covariance of mates. The reductions were larger than $75 \%$, because the predictions take account of the populations being finite. This caused a reduction in the rate of inbreeding by up to $15 \%$ (Fig. 1). 
(a)

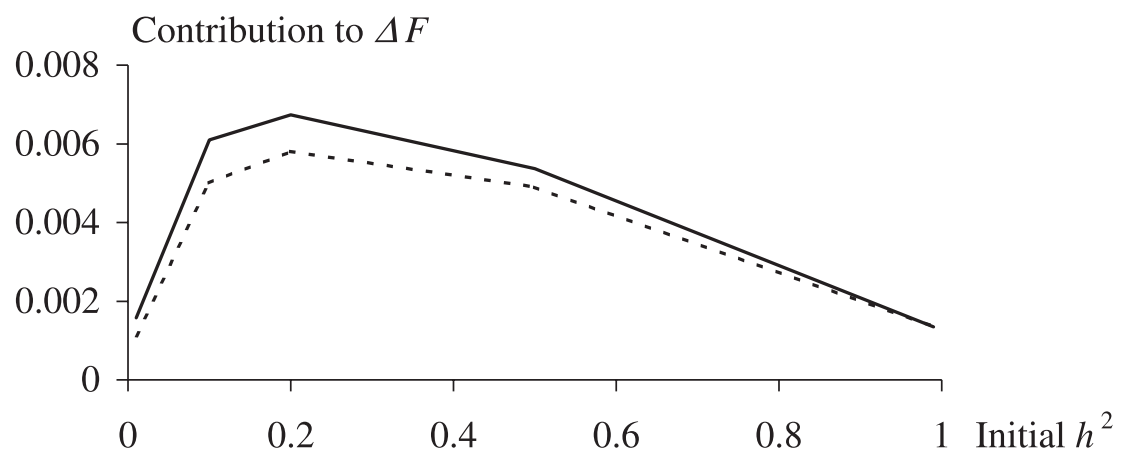

(b)

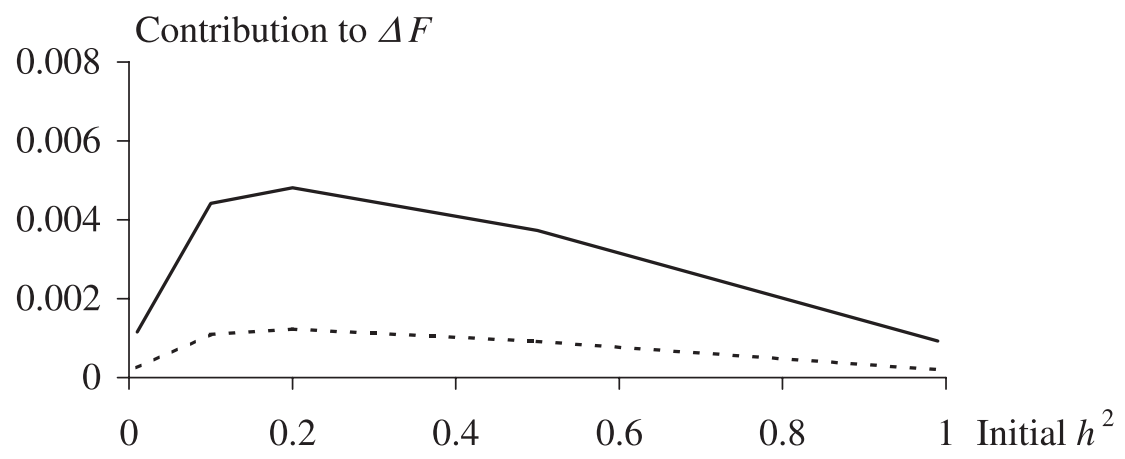

(c)

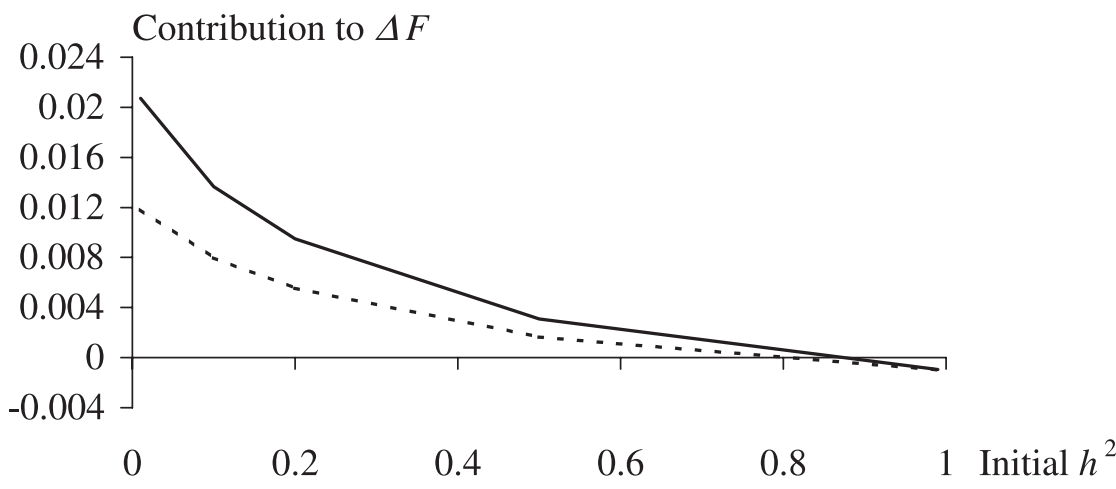

Figure 2. Contribution to the rate of inbreeding $(\Delta F)$ from (a) directional selection, (b) covariance between mates, and (c) the Poisson correction for hierarchical (full line) and factorial (dotted line) mating in an index selection scheme with 20 sires and 40 dams for a range of initial heritabilities $\left(h^{2}\right)$. 


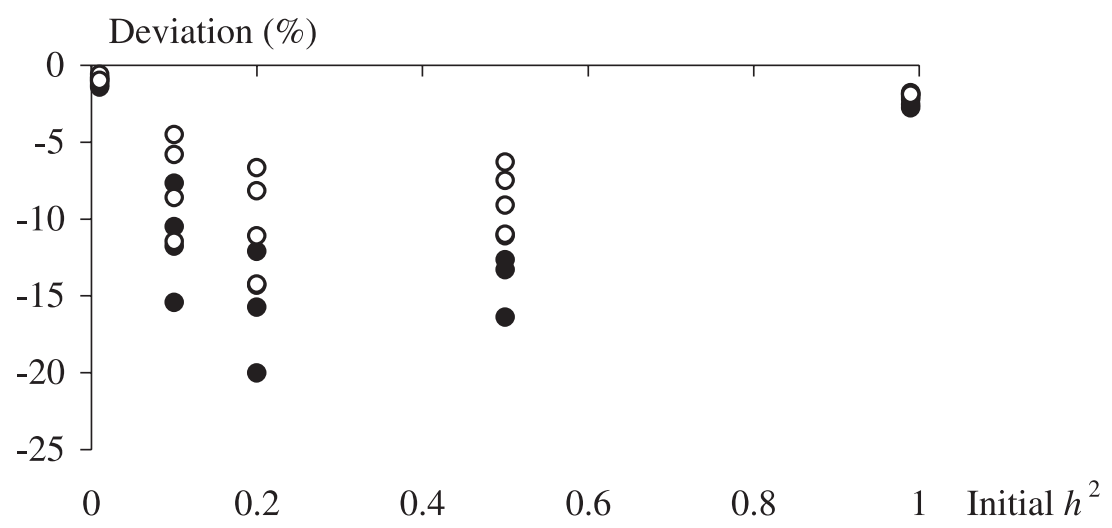

Figure 3. Deviations from linearity measured as differences (\%) between simulated rates of inbreeding and rates of inbreeding calculated using simulated parameters with a Poisson correction not accounting for non-linearity for a range of initial heritabilities $\left(h^{2}\right)$. A negative difference means that the calculated rate of inbreeding is smaller than the simulated. Results are for schemes with index selection using hierarchical (dots) or factorial (circles) mating.

\subsection{Validating the model}

\subsubsection{Rates of inbreeding}

The predictions of the rate of inbreeding were compared to results from stochastic simulations (see Fig. 1 for a specific size of breeding program). The predictions captured both the trend and the level of the rate of inbreeding over the range of heritabilities, selection criteria, and mating schemes. Under index selection, the relative prediction errors (Fig. 4a and Tab. I) were rarely larger than $10 \%$ except in the extreme cases, where the initial heritability was 0.01 .

Less than a third of the total prediction error for low to moderate heritabilities $(\leq 0.5)$ was due to errors in predicting the regression parameters (Fig. 4b). The rest of the error was due to errors in predicting the Poisson correction (Fig. 4c). The importance of these sources changed with heritability: the overprediction of the rate of inbreeding for initial heritabilities of less than 0.2 was primarily due to errors in predicting the Poisson correction, whereas the underprediction of the rate of inbreeding for an initial heritability of 0.99 was due to errors in predicting the regression parameters.

The relatively large errors (up to 20\%) in the regression parameters (Fig. 5) were translated into only small errors in the predicted rates of inbreeding (Fig. 4b). The prediction errors for the regressions on the first (Fig. 5a) and the second (Fig. 5b) selective advantages were of the same order and showed 
(a)

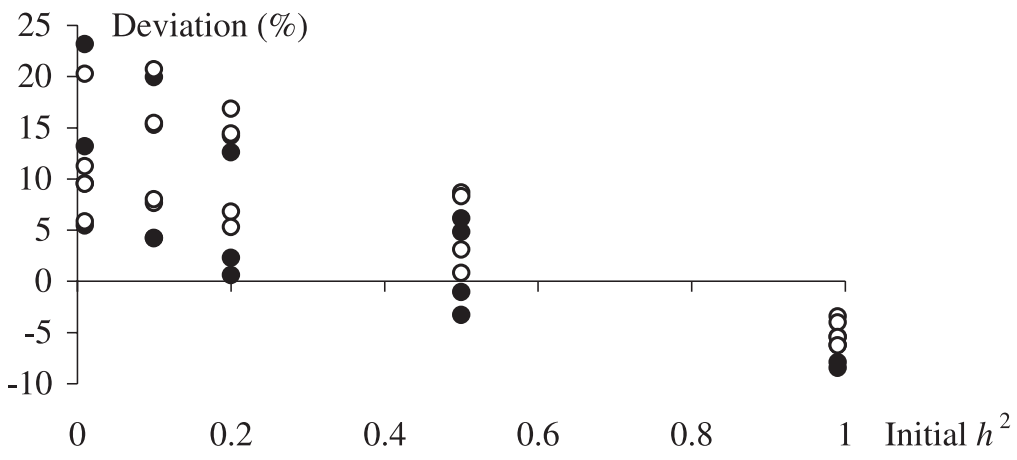

(b) 257 Deviation (\%)

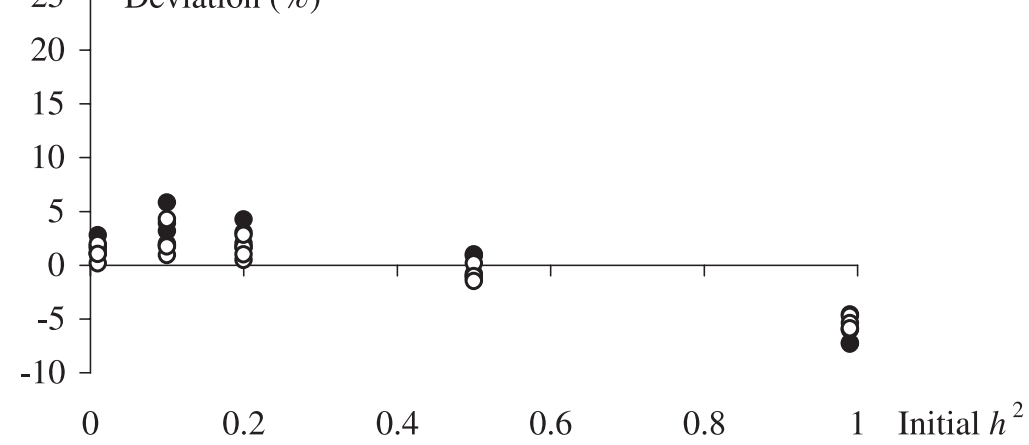

(c) 25$]$ Deviation $(\%)$

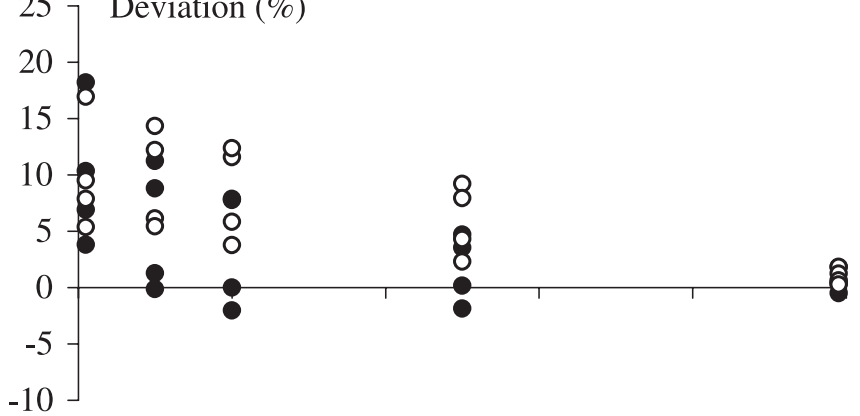
0
0.2
0.4
0.6
0.8
1 Initial $h^{2}$

Figure 4. Deviations (\%) of calculated from simulated values of rate of inbreeding for populations under index selection with hierarchical (dots) and factorial (circles) mating for a range of initial heritabilities $\left(h^{2}\right)$. Rate of inbreeding calculated with (a) all predicted values, (b) predicted regression parameters and simulated values for the rest of the terms, and (c) predicted Poisson correction and simulated values for the rest of the terms. 
Table I. Predicted $(\mathrm{p})$ and simulated $\left(_{\mathrm{s}}\right)$ rates of gain in phenotypic standard deviations $(\Delta G)$ and inbreeding $(\Delta F)$ and prediction error in percentage of the simulated rates for a number of scenarios where selection is on a sib index. $N_{m}\left(N_{f}\right)$ is the number of male (female) parents. $h_{0}^{2}$ is the initial heritability.

\begin{tabular}{|c|c|c|c|c|c|c|c|c|c|c|c|c|c|c|}
\hline \multirow[b]{2}{*}{$N_{m}$} & \multirow[b]{2}{*}{$N_{f}$} & \multirow[b]{2}{*}{$h_{0}^{2}$} & \multicolumn{4}{|c|}{ Hierarchical mating } & \multirow[b]{2}{*}{$\Delta F_{\mathrm{s}}$} & \multirow[b]{2}{*}{ error $(\%)$} & \multicolumn{6}{|c|}{ Factorial mating } \\
\hline & & & $\Delta G_{\mathrm{p}}$ & $\Delta G_{\mathrm{s}}$ & error $(\%)$ & $\Delta F_{\mathrm{p}}$ & & & $\Delta G_{\mathrm{p}}$ & $\Delta G_{\mathrm{s}}$ & error $(\%)$ & $\Delta F_{\mathrm{p}}$ & $\Delta F_{\mathrm{s}}$ & error $(\%)$ \\
\hline \multirow{3}{*}{20} & \multirow{3}{*}{40} & 0.01 & 0.024 & 0.022 & 9 & 0.0351 & 0.0311 & 13 & 0.021 & 0.020 & 4 & 0.0237 & 0.0213 & 11 \\
\hline & & 0.2 & 0.304 & 0.298 & 2 & 0.0324 & 0.0288 & 13 & 0.292 & 0.290 & 1 & 0.0230 & 0.0201 & 14 \\
\hline & & 0.5 & 0.602 & 0.599 & 1 & 0.0225 & 0.0212 & 6 & 0.596 & 0.596 & 0 & 0.0175 & 0.0161 & 9 \\
\hline \multirow{3}{*}{20} & \multirow{3}{*}{80} & 0.01 & 0.030 & 0.026 & 12 & 0.0388 & 0.0315 & 23 & 0.027 & 0.025 & 7 & 0.0292 & 0.0243 & 20 \\
\hline & & 0.2 & 0.342 & 0.335 & 2 & 0.0307 & 0.0270 & 14 & 0.333 & 0.330 & 1 & 0.0241 & 0.0207 & 17 \\
\hline & & 0.5 & 0.669 & 0.667 & 0 & 0.0199 & 0.0190 & 5 & 0.0663 & 0.665 & 0 & 0.0166 & 0.0153 & 8 \\
\hline \multirow{3}{*}{40} & \multirow{3}{*}{80} & 0.01 & 0.024 & 0.023 & 5 & 0.0171 & 0.0163 & 5 & 0.022 & 0.021 & 2 & 0.0117 & 0.0110 & 6 \\
\hline & & 0.2 & 0.307 & 0.304 & 1 & 0.0158 & 0.0155 & 2 & 0.296 & 0.295 & 0 & 0.0114 & 0.0106 & 7 \\
\hline & & 0.5 & 0.606 & 0.607 & 0 & 0.0111 & 0.0112 & -1 & 0.600 & 0.602 & 0 & 0.0086 & 0.0084 & 3 \\
\hline \multirow{3}{*}{40} & \multirow{3}{*}{160} & 0.01 & 0.030 & 0.028 & 6 & 0.0189 & 0.0173 & 10 & 0.028 & 0.027 & 4 & 0.0143 & 0.0131 & 10 \\
\hline & & 0.2 & 0.345 & 0.343 & 1 & 0.0151 & 0.0150 & 1 & 0.337 & 0.337 & 0 & 0.0119 & 0.0113 & 5 \\
\hline & & 0.5 & 0.672 & 0.674 & 0 & 0.0098 & 0.0101 & -3 & 0.667 & 0.671 & 0 & 0.0082 & 0.0081 & 1 \\
\hline
\end{tabular}

Standard errors of means of 5000 replicates of simulated rates of gain were less than $0.3 \%$ and of rates of inbreeding were less than $0.2 \%$. 
(a)

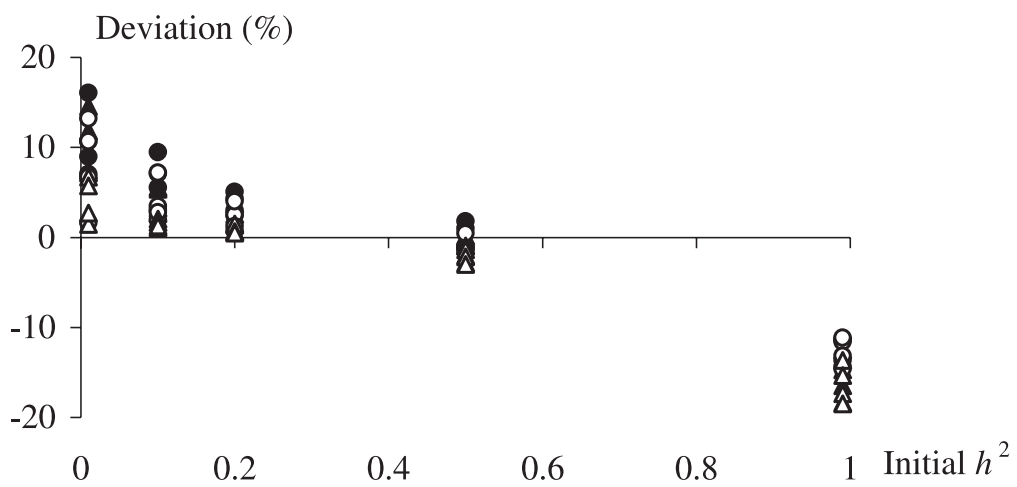

(b) Deviation (\%)

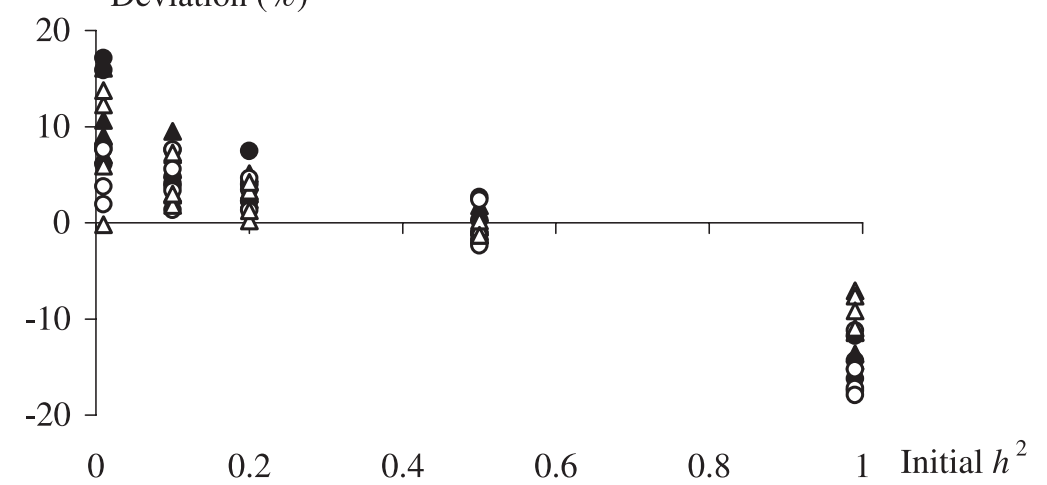

Figure 5. Deviations (\%) of predicted from simulated values of (a) regressions on the first selective advantage and (b) regressions on the second selective advantage for males (circles) and females (triangles) in populations under index selection with hierarchical (closed symbols) and factorial (open symbols) mating for a range of initial heritabilities $\left(h^{2}\right)$.

the same trend with over-predicted regressions for low heritabilities and underpredicted regressions for a very high heritability.

The errors in predicting the Poisson correction might be due to both errors in predicting the variance of family size as well as errors in capturing the non-linearity of the relationship between contributions and selective advantages. The over-prediction of the Poisson correction for an initial heritability of 0.01 was partly due to over-rating the genetic variance and the variance of the index at equilibrium (results not shown).

Under mass selection, the prediction errors of rates of inbreeding were smaller, ranging from -8 to $7 \%$ (results not shown). The errors largely arose from errors in the prediction of the regression parameters. 


\subsubsection{Rates of gain}

For a number of scenarios, the predicted and simulated rates of gain are given in Table I. The rate of gain appeared to be unaffected by the mating scheme, when mass selection was practised (results not shown). Under index selection, the rate of gain was slightly lower for schemes with factorial mating compared to hierarchical mating due to a lower accuracy of the index on which selection is based. The prediction errors of the rate of gain were only larger than $5 \%$ in the extreme case when the initial heritability was 0.01 and selection was based on the sib index. The prediction errors arose almost entirely from errors in the predicted regression parameters, whereas the deviations from linearity in the relationship between the genetic contributions and the selective advantages had very limited impact on the precision of the predicted rates of gain (results not shown). Under mass selection the prediction errors were smaller ranging from -1 to $5 \%$ (results not shown).

\section{DISCUSSION}

This study has shown that predictions of rates of inbreeding using the framework provided by expected long-term genetic contributions can reveal why factorial mating is superior to hierarchical mating in decreasing the rate of inbreeding, because the predictions (1) dissected the factors contributing to the rate of inbreeding and (2) were sufficiently precise.

Factorial mating reduces the rate of inbreeding under mass selection relative to hierarchical mating, because the variance of the second selective advantage is reduced. When viewed from a perspective of generating gain, this is a kind of error term, since it gives rise to inbreeding but not gain. The interpretation of this is that genes that are not particular good have been linked to better genes from the mate. Therefore, they "hitchhike" to higher frequencies than their merit actually warrants, and thereby generate variance of contributions without generating a positive covariance between the contribution and the Mendelian sampling term. Factorial mating reduces this error term.

The distribution of genetic contributions determines the rates of gain and inbreeding. For a given rate of inbreeding, the maximum rate of genetic gain is achieved by a specific set of contributions. These optimum contributions are a simple function of the Mendelian sampling terms [19]. However, Mendelian sampling terms are not known with certainty at the time of selection. As generations pass, the accuracies of the Mendelian sampling terms of the ancestor generation increases, and selection in the descendant generations attempts 
to change the contributions of the ancestors according to the new information. The degree to which a mating system allows realised contributions to move towards the ideal distribution will determine the success of the mating strategy, because deviations from the optimal will generate inbreeding without generating genetic gain. Factorial mating reduces these deviations relative to hierarchical mating. In other words, factorial mating reduces the confounding between male and female, allowing the optimum contribution of each individual to be more closely approached.

Optimum contribution selection aims at the optimum distribution of the genetic contributions in the first generation based on predicted Mendelian sampling terms [1]. As information on the Mendelian sampling term accumulates over generations, contributions will be changed on the way to convergence. Factorial mating reduces the covariance of contributions between mates and, thus, eases the future adjustment of the distribution of contributions and, consequently, is beneficial even with optimum contribution selection [13].

Factorial mating is also advantageous under index selection, because, most importantly, the Poisson correction is smaller. This is a consequence of the different structure of the selected population under factorial mating. There are more, but smaller, full-sib families of selection candidates where both parents have selective advantages above average compared to under hierarchical mating. This results in both smaller variance of family size and smaller deviations from linearity in the relationship between the genetic contribution and the selective advantages. This difference occurs because, with hierarchical mating, a few good ancestors that happened to be mated to other good ancestors contribute very highly to the gene pool. With factorial mating more of the good ancestors have chances to be mated to other good ancestors, whereby the contributions are more evenly distributed. An additional benefit of factorial mating is a smaller error variance, i.e. a smaller variance of those contributions that do not generate gain.

The predictions of the rate of inbreeding were sufficiently precise for most purposes. However, errors in the predictions occurred for several reasons. First, the equilibrium parameters were calculated assuming infinite population size and uncorrelated indices of selection candidates. This resulted in an over-prediction of the genetic variance for low to moderate heritabilities, especially under index selection. Second, the long-term genetic contribution was modelled using multiple linear regression on the selective advantages. The condition of linearity was not met by the contributions as they can only take on non-negative values. The linearity assumption was violated the most, when the rate of inbreeding is high, i.e. for moderate heritabilities under index 
selection. The deviations from linearity were included within the term intended to cope with the deviations from Poisson family size. Third, the predictions of the regression parameters of the linear model (1) were not perfect, because the two regression models involved in the predictions were approximate [18]. This, however, only contributed to small errors. Fourth, the calculation of deviations of variance from the variance expected under a Poisson distribution was approximate (App. C). This was the major reason for the over-prediction of the rate of inbreeding for low heritabilities with index selection. Generally, the errors were largest, in absolute sense, for unrealistic scenarios, e.g. with heritability of 0.99 and for schemes with inbreeding rates higher than $2 \%$ per generation. Thus, for realistic scenarios, the predictions were reasonably precise.

The results of this study are valid for selection on phenotype or on an index based on information from full- and half-sibs. The model used is not expected to be adequate for situations with selection on best linear unbiased predictors (BLUP), because of increased deviations from a linear relationship between the genetic contributions and selective advantages [3]. In addition, other selective advantages, e.g. higher order terms of the breeding value, could be needed [3]. However, the general point of this study, the improved family structure with factorial mating, is expected to hold even in situations with selection on BLUP.

The results of this study underline that the conscious choice of mating system should be an integral part of designing a breeding scheme. The family structure, the distribution of the genetic contributions, and therefore the rate of inbreeding, is affected by the implemented mating scheme. It is therefore obvious that other mating systems, including different kinds of non-random mating, that has been shown to affect the rate of inbreeding, also changes the distribution of the long-term genetic contributions. Further studies might reveal the underlying impact of the different mating systems on the development of the genetic contributions.

\section{ACKNOWLEDGEMENTS}

The authors gratefully acknowledge funding from the Royal Veterinary and Agricultural University, Denmark, and Department for Environment, Food, and Rural Affairs (DEFRA), UK.

\section{REFERENCES}

[1] Avendaño S., Woolliams J.A., Villanueva B., Mendelian sampling terms as the selective advantage in optimum breeding schemes with restrictions on the rate of inbreeding, Genet. Res. 83 (2004) 55-64. 
[2] Bijma P., Woolliams J.A., Prediction of genetic contributions and generation intervals in populations with overlapping generations under selection, Genetics 151 (1999) 1197-1210.

[3] Bijma P., Woolliams J.A., Prediction of rates of inbreeding in populations selected on best linear unbiased prediction of breeding value, Genetics 156 (2000) 361-373.

[4] Bijma P., van Arendonk J.A.M., Woolliams J.A., A general procedure for predicting rates of inbreeding in populations undergoing mass selection, Genetics 154 (2000) 1865-1877.

[5] Bulmer M.G., The mathematical theory of quantitative genetics, 1st edn., Clarendon Press, Oxford, 1980.

[6] Burrows P.M., Inbreeding under selection from unrelated families, Biometrics 40 (1984) 357-366.

[7] Burrows P.M., Inbreeding under selection from related families, Biometrics 40 (1984) 895-906.

[8] Fisher R.A., The correlation between relatives on the supposition of Mendelian inheritance, Trans. Roy. Soc. Edin. 52 (1918) 399-433.

[9] James J.W., McBride G., The spread of genes by natural and artificial selection in closed poultry flock, J. Genet. 56 (1958) 55-62.

[10] Mendell N.R., Elston R.C., Multifactorial qualitative traits: Genetic analysis and prediction of recurrence risks, Biometrics 30 (1974) 41-57.

[11] Nicholas F.W., Smith C., Increased rates of genetic change in dairy cattle by embryo transfer and splitting, Anim. Prod. 36 (1983) 341-353.

[12] Ruane J., The effect of alternative mating designs and selection strategies on adult multiple ovulation and embryo transfer (MOET) nucleus breeding schemes in dairy cattle, Genet. Sel. Evol. 23 (1991) 47-65.

[13] Sonesson A.K., Meuwissen T.H.E., Mating schemes for optimum contribution selection with constrained rates of inbreeding, Genet. Sel. Evol. 32 (2000) 231-248.

[14] Strandén I., Mäki-Tanila A., Mäntysaari E.A., Genetic progress and rate of inbreeding in a closed adult MOET nucleus under different mating strategies and heritabilities, J. Anim. Breed. Genet. 108 (1991) 401-411.

[15] Woolliams J.A., Modifications to MOET nucleus breeding schemes to improve rates of genetic progress and decrease rates of inbreeding in dairy cattle, Anim. Prod. 49 (1989) 1-14.

[16] Woolliams J.A., Bijma P., Predicting rates of inbreeding in populations undergoing selection, Genetics 154 (2000) 1851-1864.

[17] Woolliams J.A., Thompson R., A theory of genetic contributions, Proc. 5th World Cong. Genet. Appl. Livest. Prod., University of Guelph, 7-12 August 1994, Vol. 19, Guelph, pp. 127-134.

[18] Woolliams J.A., Bijma P., Villanueva B., Expected genetic contributions and their impact on gene flow and genetic gain, Genetics 153 (1999) 1009-1020.

[19] Woolliams J.A., Pong-Wong R., Villanueva B., Strategic optimisation of shortand long-term gain and inbreeding in MAS and non-MAS schemes, Proc. 7th World Cong. Genet. Appl. Livest. Prod., Montpellier, 19-23 August 2002, Inra, Castanet-Tolosan, CD-ROM communication 23-02. 
[20] Wray N.R., Hill W.G., Asymptotic rates of response from index selection, Anim. Prod. 49 (1989) 217-227.

[21] Wray N.R., Thompson R., Prediction of rates of inbreeding in selected populations, Genet. Res. 55 (1990) 41-54.

[22] Wray N.R., Woolliams J.A., Thompson R., Prediction of rates of inbreeding in populations undergoing index selection, Theor. Appl. Genet. 87 (1994) 878-892.

\section{APPENDIX A: PREDICTING THE REGRESSION PARAMETERS}

In the following, the genetic variance among selected parents of sex $q$ at equilibrium is defined as:

$$
\sigma_{A q}^{2}=\left(1-k_{q} r_{I A}^{2}\right) \sigma_{A}^{2}
$$

where $k_{q}$ is the variance reduction factor for parents of sex $q, r_{I A}$ is the accuracy of the selection criterion (equal for the two sexes in this study), and $\sigma_{A}^{2}$ is the equilibrium genetic variance of the population.

The two linear models describing the inheritance of selective advantages were calculated from the following vectors and matrices, which contain variances and covariances between the selective advantages of the parents and of the offspring and the selection criterion of the offspring [18]:

$\mathbf{S}_{\mathbf{q}}$, (co)variance matrix of selective advantages of parents of sex q.

$\mathbf{T}_{\mathbf{p q}}$, covariance matrix of selective advantages of offspring of sex $\mathrm{p}$ with selective advantages of parents of sex q.

$\mathbf{s}_{\mathbf{q}}$, covariance vector of selective advantages of parents of sex q with selection criterion of offspring.

$\mathbf{t}_{\mathbf{p}}$, covariance vector of selective advantages of offspring of sex $p$ with selection criterion of offspring.

$\sigma_{I}^{2}$, variance of selection criterion.

$\mathbf{S}_{\mathbf{m}}=\left[\begin{array}{cc}\left(1-\frac{1}{N_{m}}\right) \sigma_{A m}^{2} & 0 \\ 0 & \left(\frac{1}{d_{m}}-\frac{1}{N_{f}}\right) \sigma_{A f}^{2}\end{array}\right]$,

$\mathbf{S}_{\mathbf{f}}=\left[\begin{array}{cc}\left(1-\frac{1}{N_{f}}\right) \sigma_{A f}^{2} & 0 \\ 0 & \left(\frac{1}{d_{f}}-\frac{1}{N_{m}}\right) \sigma_{A m}^{2}\end{array}\right]$, 


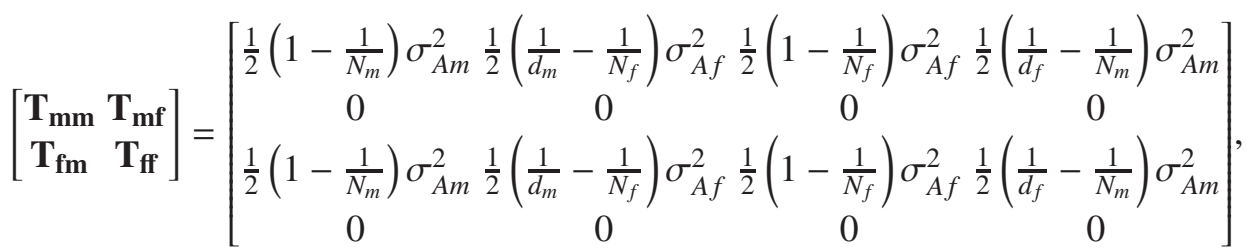

$$
\begin{aligned}
& {\left[\begin{array}{c}
\mathbf{S}_{\mathbf{m}} \\
\mathbf{S}_{\mathbf{f}}
\end{array}\right]=\left[\begin{array}{c}
\frac{1}{2}\left[c_{3}\left(1-\frac{1}{N_{m}}\right)+\frac{c_{4}-c_{2}}{d_{f}}\left(1-\frac{d_{m}}{N_{f}}\right)\right] \sigma_{A m}^{2} \\
\frac{1}{2}\left(c_{4}+c_{3}-c_{2}\right)\left(\frac{1}{d_{m}}-\frac{1}{N_{f}}\right) \sigma_{A f}^{2} \\
\frac{1}{2}\left[c_{4}\left(1-\frac{1}{N_{f}}\right)+\frac{c_{3}-c_{2}}{d_{m}}\left(1-\frac{d_{f}}{N_{m}}\right)\right] \sigma_{A f}^{2} \\
\frac{1}{2}\left(c_{4}+c_{3}-c_{2}\right)\left(\frac{1}{d_{f}}-\frac{1}{N_{m}}\right) \sigma_{A m}^{2}
\end{array}\right],} \\
& {\left[\begin{array}{c}
\mathbf{t}_{\mathbf{m}} \\
\mathbf{t}_{\mathbf{f}}
\end{array}\right]=\left[\begin{array}{c}
\left(1-\frac{1}{N_{m}}\right) \sigma_{I}^{2} \\
0 \\
\left(1-\frac{1}{N_{f}}\right) \sigma_{I}^{2} \\
0
\end{array}\right] \text {. }}
\end{aligned}
$$

From the matrices above, the matrices of regression parameters for the two models, $\boldsymbol{\Lambda}$ and $\boldsymbol{\Pi}$, were calculated sub-matrix by sub-matrix using the following algorithm:

1. Set $\mathrm{q}=$ male parents, subscript $m$.

2. Calculate the 1 by 2 vector $\mathbf{w}_{\mathbf{q}}=\mathbf{s}_{\mathbf{q}}^{\mathbf{T}} \mathbf{S}_{\mathbf{q}}^{-1}$.

3. Set $\mathrm{p}=$ male offspring, subscript $m$.

4. Calculate the 1 by 2 vector $\lambda_{\mathbf{p q}}=\frac{i_{p}}{\sigma_{I}} \mathbf{w}_{\mathbf{q}}$.

5. Calculate the 2 by 2 matrix $\boldsymbol{\pi}_{\mathbf{p q}}=\left(\mathbf{T}_{\mathbf{p q}}-\frac{k_{p}}{\sigma_{I}^{2}} \mathbf{t}_{\mathbf{p}} \mathbf{S}_{\mathbf{q}}^{\mathbf{T}}\right) \mathbf{S}_{\mathbf{q}}^{-\mathbf{1}}$.

6. Repeat 4. and 5. for $\mathrm{p}=$ female offspring, subscript $f$.

7. Repeat 2. to 6 . for $\mathrm{q}=$ female parents, subscript $f$.

8. Construct $\boldsymbol{\Lambda}=\left[\begin{array}{cc}\lambda_{\mathrm{mm}} & \lambda_{\mathrm{mf}} \\ \lambda_{\mathrm{fm}} & \lambda_{\mathrm{ff}}\end{array}\right]$, which is a 2 by 4 matrix.

9. Construct $\Pi=\left[\begin{array}{cc}\pi_{\mathrm{mm}} & \pi_{\mathrm{mf}} \\ \pi_{\mathrm{fm}} & \boldsymbol{\pi}_{\mathrm{ff}}\end{array}\right]$, which is a 4 by 4 matrix.

Then the vector of $\beta$ 's was calculated using these matrices:

$$
\boldsymbol{\beta}=\mathbf{N}^{-1}\left(\mathbf{I}-\frac{1}{2} \boldsymbol{\Pi}^{\mathbf{T}}\right)^{-1}\left(\frac{1}{2} \boldsymbol{\Lambda}^{\mathbf{T}}\right)\left[\begin{array}{c}
\frac{1}{2} \\
\frac{1}{2}
\end{array}\right]
$$

where $\mathbf{N}=\operatorname{diag}\left(N_{m}, N_{m}, N_{f}, N_{f}\right)$, and $\mathbf{I}$ is a 4 by 4 identity matrix. 
The $\beta$ 's quantify the effect of selective advantages on the long-term genetic contributions of selected animals of a certain sex. The selective advantages are all things affecting this contribution. Therefore, in calculating the regression coefficients only the selective advantages of the given sex were considered and covariances between selective advantages of male and female parents were ignored. As a result, the $\lambda$ 's and $\pi$ 's were calculated as regressions on the selective advantages of parents separately for male and female parents in step 4 and 5. Consequently, the $\lambda$ 's quantify the effect of the selective advantages on the selection criteria of the offspring. And the $\pi$ 's quantify the effect of the selective advantages on the selective advantages of the offspring. In this way, the gene flow of an individual is calculated based on the selective advantages, and the rate of inbreeding is calculated from the sum of squared expectations of gene flow over individuals.

\section{APPENDIX B: EXPECTATIONS, VARIANCES, AND COVARIANCES}

The expectations, variances, and covariances in equations (2) and (4) were calculated using standard index theory and given in Table B.I. The regression of the index on the Mendelian sampling term, $\tau_{w}$, which was used in the parameters below, were derived from the index weights, $c_{1}$ to $c_{4}$ :

$$
\tau_{w}=c_{1}+\left(c_{2}-c_{1}\right) \frac{1}{n}+\left(c_{3}-c_{2}\right) \frac{1}{n d_{m}}+\left(c_{4}-c_{2}\right) \frac{1}{n d_{f}}
$$

where $n$ is the number of offspring per mating, $d_{m}$ is the number of dams mated to each sire, and $d_{f}$ is the number of sires mated to each dam.

Using equal weights of the information sources resulted in mass selection in which case $\tau_{w}=h^{2}, \sigma_{I}=h \sigma_{A}, r_{I A}^{2}=h^{2}$. In this case, the formulas in the right column collapsed to the ones in the middle column. This provided a check, that the (co)variances of the information sources were calculated correctly.

\section{APPENDIX C: PREDICTING THE POISSON CORRECTION}

This appendix describes the calculation of the correction of the rate of inbreeding due to deviation of family sizes after selection from a Poisson distribution. The calculation followed Woolliams and Bijma [16], but was extended to be applied to factorial mating.

For $q=$ male or female parents in equation (4) $\delta_{q}=\alpha^{\mathbf{T}} \mathbf{V}_{\mathbf{n}(\mathbf{q}) \text {,dev }} \boldsymbol{\alpha}$, where $\boldsymbol{\alpha}^{\mathbf{T}}=\left[\begin{array}{ll}\alpha_{m} & \alpha_{f}\end{array}\right]$, and $\mathbf{V}_{\mathbf{n}(\mathbf{q}) \text {,dev }}$ is a 2 by 2 matrix with (co)variance deviations 
Table B.I. List of expectations, covariances, and variances used in equations (2) and (4) calculated from standard index theory.

\begin{tabular}{lcc}
\hline Parameter & Mass selection & Index selection \\
\hline $\mathrm{E}\left[a_{i(q)} \mid i\right.$ is selected $]$ & $\frac{1}{2} \sigma_{A, 0}^{2} i_{q} \sigma_{P}^{-1}$ & $\frac{1}{2} \sigma_{A, 0}^{2} i_{q} \tau_{w} \sigma_{I}^{-1}$ \\
$\operatorname{Cov}\left(s_{i(q), 1}-\bar{s}_{q, 1} ; a_{i(q)}\right)$ & $\frac{1}{2}\left(1-\frac{1}{N_{q}}\right)\left(1-k_{q} h^{2}\right) \sigma_{A, 0}^{2}$ & $\frac{1}{2}\left(1-\frac{1}{N_{q}}\right)\left(1-k_{q} \tau_{w}\right) \sigma_{A, 0}^{2}$ \\
$\operatorname{Cov}\left(s_{i(q), 2}-\bar{s}_{q, 2} ; a_{i(q)}\right)$ & 0 & 0 \\
$\operatorname{Var}\left(s_{i(q), 1}-\bar{s}_{q, 1}\right)$ & $\left(1-\frac{1}{N_{q}}\right)\left(1-k_{q} h^{2}\right) \sigma_{A}^{2}$ & $\left(1-\frac{1}{N_{q}}\right)\left(1-k_{q} r_{I A}^{2}\right) \sigma_{A}^{2}$ \\
$\operatorname{Var}\left(s_{i(m), 2}-\bar{s}_{m, 2}\right)$ & $\left(\frac{1}{d_{m}}-\frac{1}{N_{f}}\right)\left(1-k_{f} h^{2}\right) \sigma_{A}^{2}$ & $\left(\frac{1}{d_{m}}-\frac{1}{N_{f}}\right)\left(1-k_{f} r_{I A}^{2}\right) \sigma_{A}^{2}$ \\
$\operatorname{Var}\left(s_{i(f), 2}-\bar{s}_{f, 2}\right)$ & $\left(\frac{1}{d_{f}}-\frac{1}{N_{m}}\right)\left(1-k_{m} h^{2}\right) \sigma_{A}^{2}$ & $\left(\frac{1}{d_{f}}-\frac{1}{N_{m}}\right)\left(1-k_{m} r_{I A}^{2}\right) \sigma_{A}^{2}$ \\
\hline
\end{tabular}

$\sigma_{A, 0}^{2}$ is the genetic variance in the unselected base population; $\sigma_{A}^{2}$ is the genetic variance under Bulmer equilibrium; $\sigma_{P}$ is the equilibrium phenotypic standard deviation; $\sigma_{I}$ is the equilibrium standard deviation of the index; $\tau_{w}$ is the regression of the index on the Mendelian sampling term; $h^{2}$ is the equilibrium heritability; $k_{q}$ is the variance reduction factor for parents of sex $q$; and $r_{I A}$ is the equilibrium accuracy of the index.

from independent Poisson variances of the number of male and female offspring selected. This ignored the differences in the expected long-term genetic contributions of the offspring, because only the overall contribution for each sex, i.e. $\alpha_{q}$, is considered. This, however, only leads to small errors [3].

In order to derive the variance deviations, the probability of co-selection of sibs was quantified by the value of $R\left(p_{m}, p_{f}, \rho\right)$, which gives the ratio of the probability under no correlation between candidates and the probability, when the correlation equals $\rho . R\left(p_{m}, p_{f}, \rho\right)$ was approximated using formula (3.4) of Mendell and Elston [10] and following Wray et al. [22]:

$$
R\left(p_{m}, p_{f}, \rho\right) \approx \frac{p_{f}}{\Phi\left[\left(i_{m} \rho-v_{f}\right)\left(1-k_{m} \rho^{2}\right)^{-\frac{1}{2}}\right]}
$$

where $p_{r}, i_{r}, k_{r}$, and $v_{r}$ are the selected proportion, the selection intensity, the variance reduction factor, and the standardised truncation point of sex $r$, respectively. $\rho$ is the correlation between selection criteria of the candidates for selection.

(C.1) gives the ratio for co-selection of sibs of different sex. For co-selection of sibs of the same sex, the selection parameters were all derived from the selected proportion of that sex. There are different $R$-values for different 
relationships, i.e. full sibs, paternal half sibs, and maternal half sibs, depending on the correlation between the candidates of interest.

The elements of the matrices of (co)variance deviations, $\mathbf{V}_{\mathbf{n}(\mathbf{q}) \text {,dev }}$, are the part of the variance of family size not accounted for by the linear model (1). They were derived using factorial hypergeometric sampling moments following the approximate approach of Burrows [6,7] and Woolliams and Bijma [16]. The following parameters were used: $n_{r}$ is the number of offspring of sex $r$ per mating, in this study $n_{m}=n_{f}, N_{q}$ is the number of parents of sex $q, \mathrm{~T}$ is the number of candidates available for selection of each sex, $d_{m}$ is the number of dams mated to each sire, and $d_{f}$ is the number of sires mated to each dam, $\rho_{F S}$ and $\rho_{P H S}$ are correlations of selection criteria of full-sibs and paternal halfsibs, respectively, and $\lambda_{i j}$ is the element in the $i$ th row and $j$ th column of $\boldsymbol{\Lambda}$.

For male parents, the number of selected offspring of sex $r$ from a family with sire $i, n_{i *}(r)$, is considered. The first diagonal element is:

$$
\begin{aligned}
& \operatorname{Var}\left[n_{i *}(m)\right]-E\left[n_{i *}(m) \mid \text { selective advantages of } i\right] \\
& =E\left[n_{i *}(m) \cdot\left(n_{i *}(m)-1\right)\right]-E\left[n_{i *}(m) \mid \text { selective advantages of } i\right]^{2} \\
& \approx\left[\frac{d_{m}\left(1-\frac{1}{n_{r}}\right)}{R\left(p_{m}, p_{m}, \rho_{F S}\right)}+\frac{d_{m}\left(d_{m}-1\right)}{R\left(p_{m}, p_{m}, \rho_{P H S}\right)}\right]\left[\frac{n_{r}^{2} N_{m}\left(N_{m}-1\right)}{T(T-1)}\right] \\
& -\left(1+\lambda_{11}^{2} \operatorname{Var}\left(s_{i(m), 1}-\bar{s}_{m, 1}\right)+\lambda_{12}^{2} \operatorname{Var}\left(s_{i(m), 2}-\bar{s}_{m, 2}\right)\right)
\end{aligned}
$$

and the second diagonal element is:

$\operatorname{Var}\left[n_{i *}(f)\right]-E\left[n_{i *}(f) \mid\right.$ selective advantages of $\left.i\right]$

$$
\begin{aligned}
& =E\left[n_{i *}(f) \cdot\left(n_{i *}(f)-1\right)\right]-E\left[n_{i *}(f) \mid \text { selective advantages of } i\right]^{2} \\
& \approx\left[\frac{d_{m}\left(1-\frac{1}{n_{r}}\right)}{R\left(p_{f}, p_{f}, \rho_{F S}\right)}+\frac{d_{m}\left(d_{m}-1\right)}{R\left(p_{f}, p_{f}, \rho_{P H S}\right)}\right]\left[\frac{n_{r}^{2} N_{f}\left(N_{f}-1\right)}{T(T-1)}\right] \\
& \quad-\left(\frac{d_{m}}{d_{f}}\right)^{2}\left(1+\lambda_{21}^{2} \operatorname{Var}\left(s_{i(m), 1}-\bar{s}_{m, 1}\right)+\lambda_{22}^{2} \operatorname{Var}\left(s_{i(m), 2}-\bar{s}_{m, 2}\right)\right) .
\end{aligned}
$$


The off-diagonal element is:

$\operatorname{Cov}\left[n_{i *}(m) ; n_{i *}(f)\right]$

$=E\left[n_{i *}(m) \cdot n_{i *}(f)\right]-E\left[n_{i *}(m) \mid\right.$ selective advantages of $\left.i\right] E\left[n_{i *}(f)\right.$

| selective advantages of $i]$

$$
\begin{aligned}
& \approx\left[\frac{d_{m}}{R\left(p_{m}, p_{f}, \rho_{F S}\right)}+\frac{d_{m}\left(d_{m}-1\right)}{R\left(p_{m}, p_{f}, \rho_{P H S}\right)}\right]\left[\frac{n_{r}^{2} N_{m} N_{f}}{T^{2}}\right] \\
& -\left(\frac{d_{m}}{d_{f}}\right)\left(1+\lambda_{11} \cdot \lambda_{21} \cdot \operatorname{Var}\left(s_{i(m), 1}-\bar{s}_{m, 1}\right)+\lambda_{12} \cdot \lambda_{22} \cdot \operatorname{Var}\left(s_{i(m), 2}-\bar{s}_{m, 2}\right)\right) .
\end{aligned}
$$

For female parents, the number of selected offspring of sex $r$ from a family with dam $j$ is considered, and the terms corresponding to the above were calculated using parameters applying to female parents.

If $d_{f}=1$, i.e. hierarchical mating, the terms involving maternal half sibs disappear, because these contain the expression $\left(d_{f}-1\right)$. If $d_{f}=1$ and $N_{m}=N_{f}$, then $d_{m}=1$ and the terms involving paternal half sibs disappear, because these contain the expression $\left(d_{m}-1\right)$.

To access this journal online: www.edpsciences.org 
